\title{
Listening to Music “in" the Library
}

\section{by Lucinda Johnston}

\begin{abstract}
Digital and streaming audio and video $(A / V)$ content have usurped the primacy of physical media materials and their playback technology within institutional music libraries, notwithstanding throwbacks to and resurgences of physical media in commercial and personal contexts. Music libraries are challenged with the conflicting responsibilities of maintaining legacy format materials that are not digitally available, continuing to collect physical resources that are not available either digitally or through institutional streaming subscriptions, and acquiring born-digital and digitized resources. They must also reconcile these responsibilities with the fact that many streaming $A / V$ resources are freely available to individual consumers. In an era of $d$ windling resources and appreciation for curated music collections, how will libraries ensure that their $A / V$ resources, in all formats, remain relevant to current and future users? This paper presents the results of an A/V usage survey administered to affiliates of the University of Alberta's Music Department to learn about the attitudes, preferences and experiences of music library users' practises for accessing recorded music.
\end{abstract}

Lucinda Johnston (lucinda.johnston@ualberta.ca) is the Liaison and Instructional Librarian at the University of Alberta Libraries.

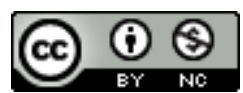

This work is licensed under a Creative Commons Attribution-NonCommercial 4.0 International License. 


\section{Background}

In August 2018, I embarked on a new profession with my appointment to the position of Music Liaison Librarian at the University of Alberta Library (UAL). Though no stranger to the world of music, I was nervous about my ability to meet the demands of my new job, which included helping departmental constituents access library resources, and supporting the UAL's Collections Strategies Unit (CSU) to make informed decisions on approval plans, streaming collections, database access and various purchases. Like any freshly minted, eager, uninitiated information professional, I felt completely ill-equipped for the task, and so immediately enrolled in professional development courses.

One of the courses I took was the ALA and MLA joint offering "Music Collection Development for Every Librarian," taught by John Redford (Biola University), Lisa Hooper (Tulane University), and CAML's very own Houman Behzadi (McGill University). The course provided instruction on the essentials of collection management from a music perspective, including a section highlighting the many challenges associated with providing access to evolving and legacy media formats. Reflecting on what I was learning in the context of the UAL's current collection development policies and access/circulation models (which had been undergoing significant changes several years immediately preceding my arrival), I knew that I needed to actively seek out more information about my users' needs and preferences if I was going to support them and the CSU effectively.

With input from several of my colleagues, ${ }^{1}$ I created a music listening survey for all staff, faculty, sessional instructors and students (graduate and undergraduate) associated with the Music Department (MD) at the University of Alberta (UA) to determine:

1. If the UAL was providing appropriate resources and services to meet their music listening/viewing needs and preferences, and

2. How the UAL can add to or improve the resources and services we do offer.

I sent out the survey to 389 recipients on April 11, 2019 via the MD's undergraduate, graduate and staff/faculty list-serves. A total of 53 responses were received between April 11 and April 26, when I turned the survey off from receiving any more responses.

\section{Survey Description}

The Music Listening Survey (see Appendix A) contained a total of eight questions divided into three sections. Section 1 - "How do you listen to music" - had three multiple-choice questions that asked respondents to classify themselves according to their status in the music department, and to

\footnotetext{
${ }^{1}$ Many thanks to the UAL librarians Lindsay Johnston, Cam LaForest and Sarah Polkinghorne for all of their feedback and support with this project.
} 
identify all music formats ${ }^{2}$ and music playback equipment that they regularly use. Legacy formats and related playback equipment referred to vinyl records (any size), cassette tapes, CDs, and DVDs. Non-legacy formats and playback equipment referred to digital audio/video files (e.g. .mp3, .mp4, .wav), streaming audio/video (e.g. Spotify, Naxos), and broadcast audio/video. Both formats and playback equipment included an "other" option.

Section 2 - "Library music resources" - gathered information about how respondents used the various music formats and non-circulating playback equipment available through the $\mathrm{UAL}^{3}$ and also had three questions. The first two multiple-choice questions asked respondents to indicate their awareness and use of available formats and playback equipment. The third, long-answer question asked respondents to explain why they have not been using formats and equipment they are aware the library provides access to.

Section 3 - "Your Feedback" - asked respondents two questions to gather information to inform future collection development and service offerings. The first multiple-choice question asked respondents to rate how important it was overall for them to be able to access specific music formats, circulating playback equipment ${ }^{4}$ and group listening space $(\mathrm{s}) .{ }^{5}$ In the final question, respondents were asked to provide any further feedback they wished to include.

In order to make filling out the survey as simple as possible for respondents, ${ }^{6}$ no survey questions were required to be answered and respondents were not restricted to only one response. Removing required responses allowed respondents to complete the survey even if they didn't want to answer a particular question. Not restricting respondents to one response meant that they did not have to log in with their UA credentials and had immediate access to the survey. Although these design choices allowed for the possibility of partial responses, I was more willing to risk gathering incomplete data than I was of gathering insufficient data overall.

\footnotetext{
${ }^{2}$ I specifically used the term "music", rather than sound or audio, because a) some of the formats, e.g., DVDs, digital files and streaming content include video components, and b) I wanted people to think about how they listen to and/or experience music specifically (as opposed to other sound events). I also used the term "formats", as opposed to recordings, because I wanted to differentiate mediums.

${ }^{3}$ The UAL currently does not circulate any playback equipment; we do circulate laptops with VLC media player installed, but they do not have CD or DVD drives and they are not promoted as "playback equipment".

${ }^{4}$ I did not ask respondents to rate the importance of non-circulating playback equipment that the UAL provides, as I believed the usage information results would imply the level of importance associated with this equipment.

${ }^{5}$ This item was included due to conversations I had with faculty in the MD who lamented that streaming audio has created an individual siloed listening culture but believed that listening to music as a group remains an important pedagogical activity, which is increasingly more difficult to create in a classroom setting.

${ }^{6}$ This survey was sent out at the end of the semester and would have been competing with concurrent requests for course and instructor feedback.
} 


\section{Survey Results}

Section 1 - How do you listen to music: "Who are you? What audio formats do you listen to? What equipment do you use?"

\section{Q1) Respondent Populations}

At the time the survey was distributed, the MD had 274 undergraduate students and 55 graduate students enrolled in music courses. The MD also had 60 full- and part-time staff (including sessional instructors) and faculty. The 53 responses that were received, from a possible 389, represented $13.6 \%$ of the total MD population. Table 1 summarizes the responses received.

Table 1: Survey response rates organized by MD population

\begin{tabular}{|l|lll|}
\hline \multicolumn{1}{|l}{ MD Population type } & $\begin{array}{l}\text { Total population } \\
\text { (\% of Total) }\end{array}$ & $\begin{array}{l}\text { Survey respondents } \\
\text { (\% of Total) }\end{array}$ & $\begin{array}{l}\text { \% Respondents compared } \\
\text { to total population }\end{array}$ \\
\hline Undergraduate Students & $274(70.4 \%)$ & $23(43.4 \%)$ & $8.4 \%$ \\
Graduate Students & $55(14.1 \%)$ & $12(22.6 \%)$ & $21.8 \%$ \\
Staff/Faculty & $60(15.4 \%)$ & $18(34 \%)$ & $30 \%$ \\
\hline Total MD Population & 389 & 53 & $13.6 \%$ \\
\hline
\end{tabular}

Undergraduate students comprise the majority of the total MD population (70.4\%) and had the highest response rate (43.4\%) compared to graduate students (22.6\%) and staff/faculty (34\%). However, the number of undergraduate respondents represented only $8.4 \%$ of the total undergraduate student population, compared with the representation of the total graduate $(21.8 \%)$ and staff/faculty (30\%) populations. The disparity in representation of the individual populations, combined with the low, overall response rate will affect how broadly we may interpret the findings from the survey.

Some respondents did not provide responses for each line in questions 4, 5 and 7 . The calculations presented in this section are based on the number of responses for each data point; all data points had between 51-53 responses.

\section{Q2) Regularly Used Music Formats}

Unsurprisingly, digital files and streaming content were the most regularly used music formats overall, and the legacy formats (vinyl, cassettes and DVDs) were the least regularly used. CDs and 
broadcast audio/video formats received higher usage than expected. Figure 1 provides a summary of respondent's overall use of the various types of music formats.

Figure 1: Percentage of overall responses, regularly used music formats

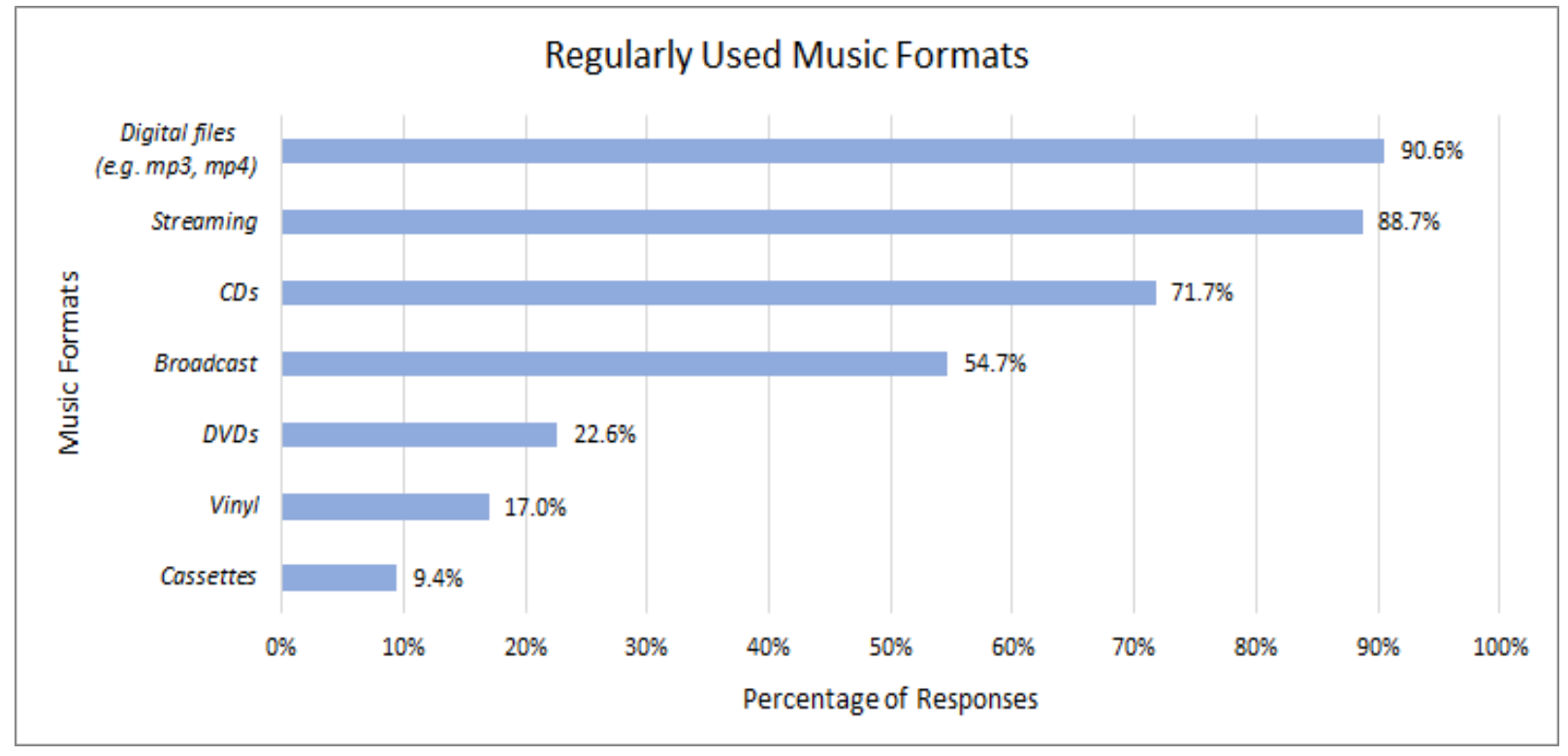

Table 2 outlines how much each population uses each music format. Legacy format (cassettes, vinyl, DVD and CDs) usage overall was dominated by staff/faculty. Non-legacy music formats (broadcast, streaming and digital files) were consistently more highly used by undergraduate and graduate students (approximately $2 / 3$ of all non-legacy music format usage).

Table 2: Music format usage by respondent population

\begin{tabular}{|l|rrrr|rrr|}
\hline \multicolumn{7}{|c|}{} & \multicolumn{9}{c|}{ Legacy Formats } & & Non-legacy formats \\
\hline Population & $\begin{array}{r}\text { Cassettes } \\
\text { (\% of Total) }\end{array}$ & $\begin{array}{r}\text { Vinyl } \\
\text { (\% of Total) }\end{array}$ & $\begin{array}{r}\text { DVDs } \\
\text { (\% of Total) }\end{array}$ & $\begin{array}{r}\text { CDs } \\
\text { (\% of Total) }\end{array}$ & $\begin{array}{r}\text { Broadcast } \\
\text { (\% of Total) }\end{array}$ & $\begin{array}{r}\text { Streaming } \\
\text { (\% of Total) }\end{array}$ & $\begin{array}{r}\text { Digital files } \\
\text { (\% of Total) }\end{array}$ \\
$\begin{array}{l}\text { Undergrads } \\
(n=23)\end{array}$ & $1(20 \%)$ & $2(22.2 \%)$ & $1(7.7)$ & $15(38.5 \%)$ & $12(41.4 \%)$ & $22(46.8 \%)$ & $21(43.8 \%)$ \\
$\begin{array}{l}\text { Graduates } \\
(n=12)\end{array}$ & $1(20 \%)$ & $2(22.2 \%)$ & $1(7.7)$ & $6(15.4 \%)$ & $6(20.7 \%)$ & $10(21.3 \%)$ & $10(20.8 \%)$ \\
$\begin{array}{l}\text { Staff/Faculty } \\
(n=20)\end{array}$ & $3(60 \%)$ & $5(55.6 \%)$ & $11(84.6)$ & $18(46.2 \%)$ & $11(37.9 \%)$ & $15(31.9 \%)$ & $17(35.4 \%)$ \\
$\begin{array}{l}\text { Total } \\
\text { Respondents }\end{array}$ & $\mathbf{5}$ & $\mathbf{9}$ & $\mathbf{1 3}$ & $\mathbf{3 9}$ & $\mathbf{2 9}$ & $\mathbf{4 7}$ & $\mathbf{4 8}$ \\
\hline
\end{tabular}




\section{Q3) Regularly used playback equipment}

As expected, the results for the regularly used playback equipment corresponded very closely to the responses for regularly used music formats, as outlined in Figure 2.

Figure 2: Percentage of overall responses, regularly used music formats

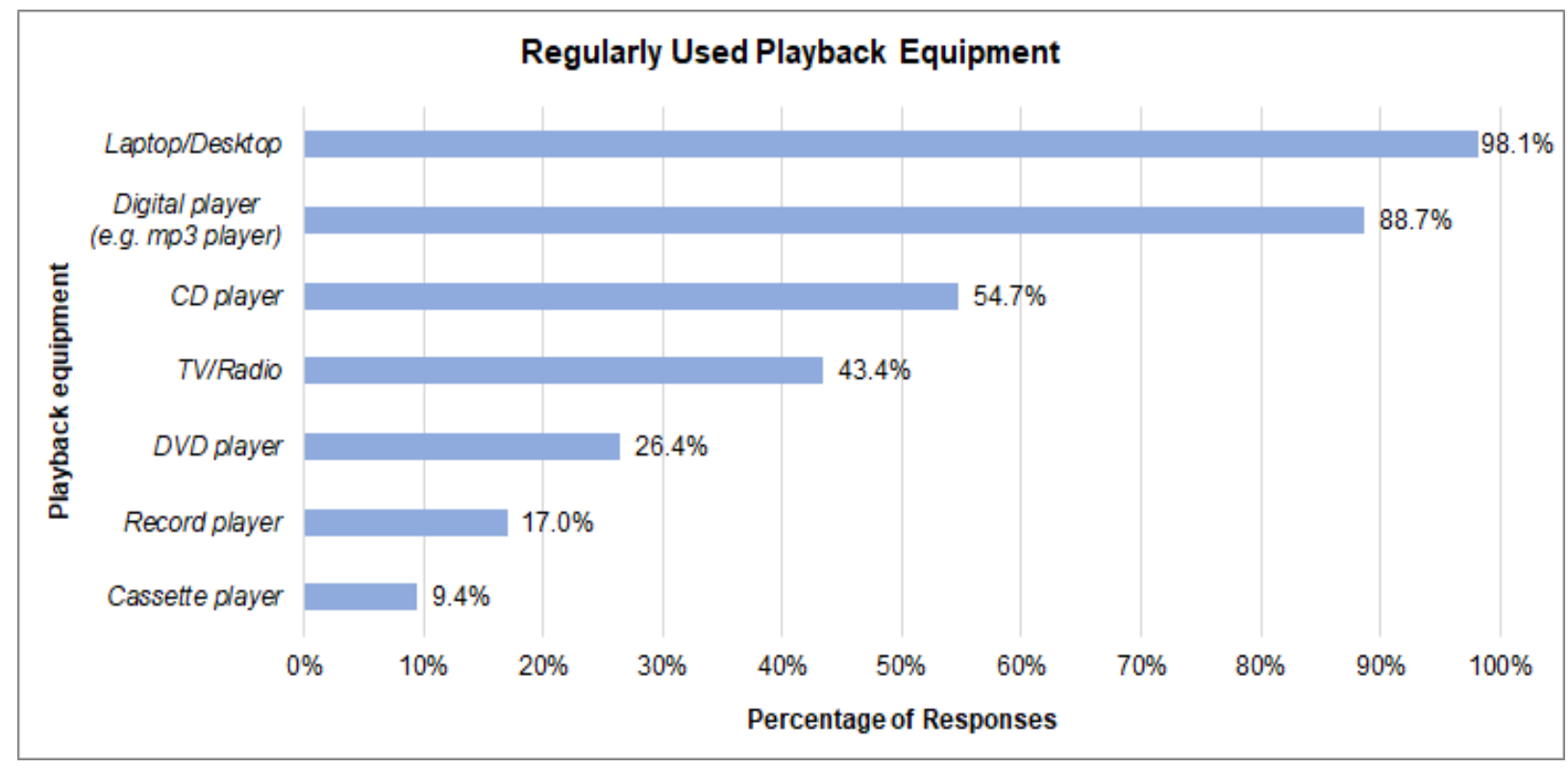

Section 2 - Library music resources: "What UAL music resources and playback equipment do you know about? Do you use them? Why not?"

\section{Q4) Experiences with music formats available at UAL}

Respondents' awareness and use of music formats are represented in Figure 3. For legacy formats (vinyl, CDs and DVDs), approximately half of the respondents (i.e., ranging from 43-68\%) were aware of them but didn't use them. Of the respondents who occasionally or regularly used these legacy formats, staff/faculty represented at least half of the respondents. Only one undergraduate respondent indicated they had occasionally used vinyl, and none have ever used DVDs. Streaming content is the only format that was regularly used equally between staff/faculty, graduate students and undergraduate students. 
Figure 3: Count of respondents' awareness and use of library music formats

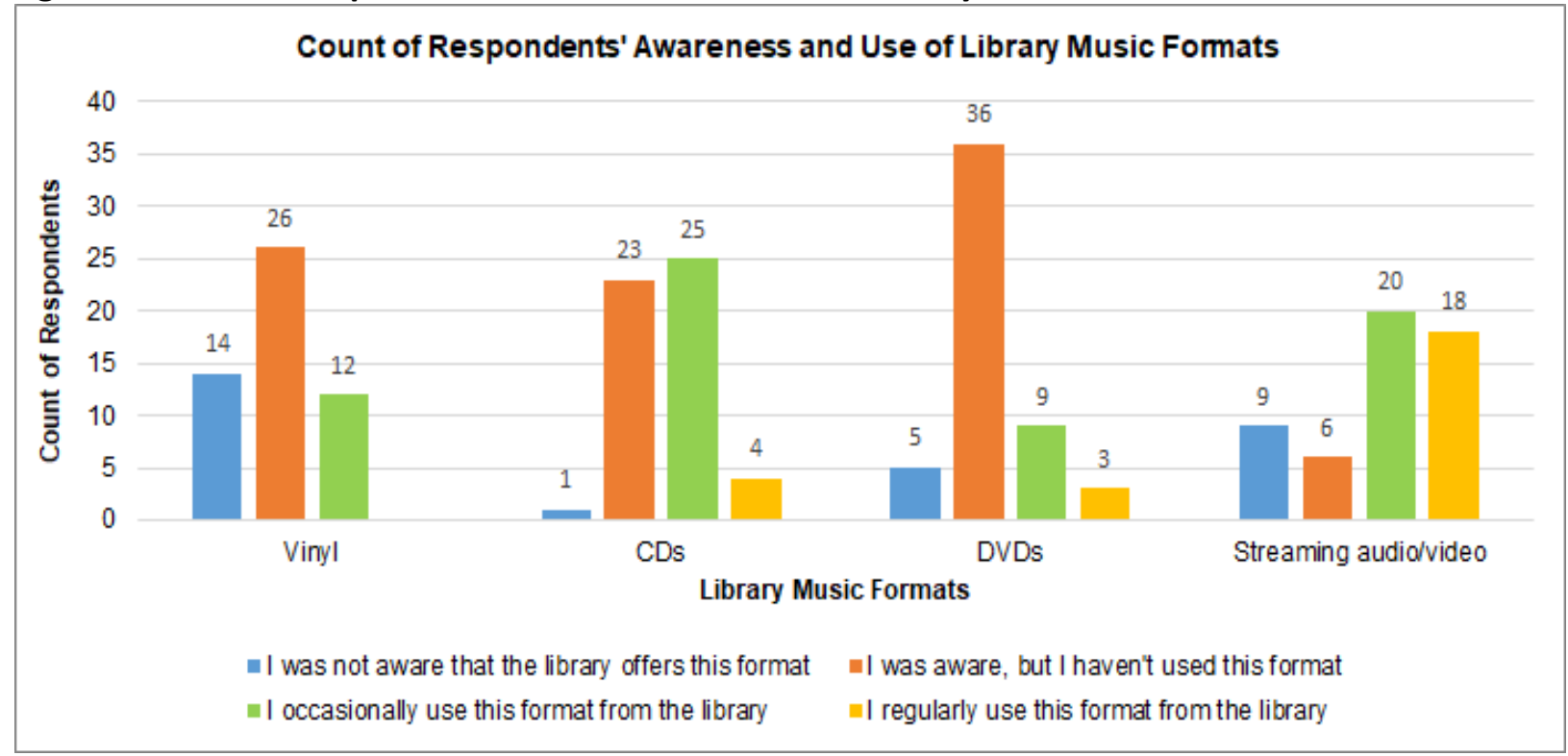

Q5) Experiences with playback equipment available at UAL

Playback equipment provided by the library was heavily underused by all respondents. Most of the occasional use of all equipment was by staff/faculty; graduate and undergraduate students indicated regular use of desktop computers, but less use of other equipment.

\section{Figure 4: Count of respondents' awareness and use of library playback equipment}

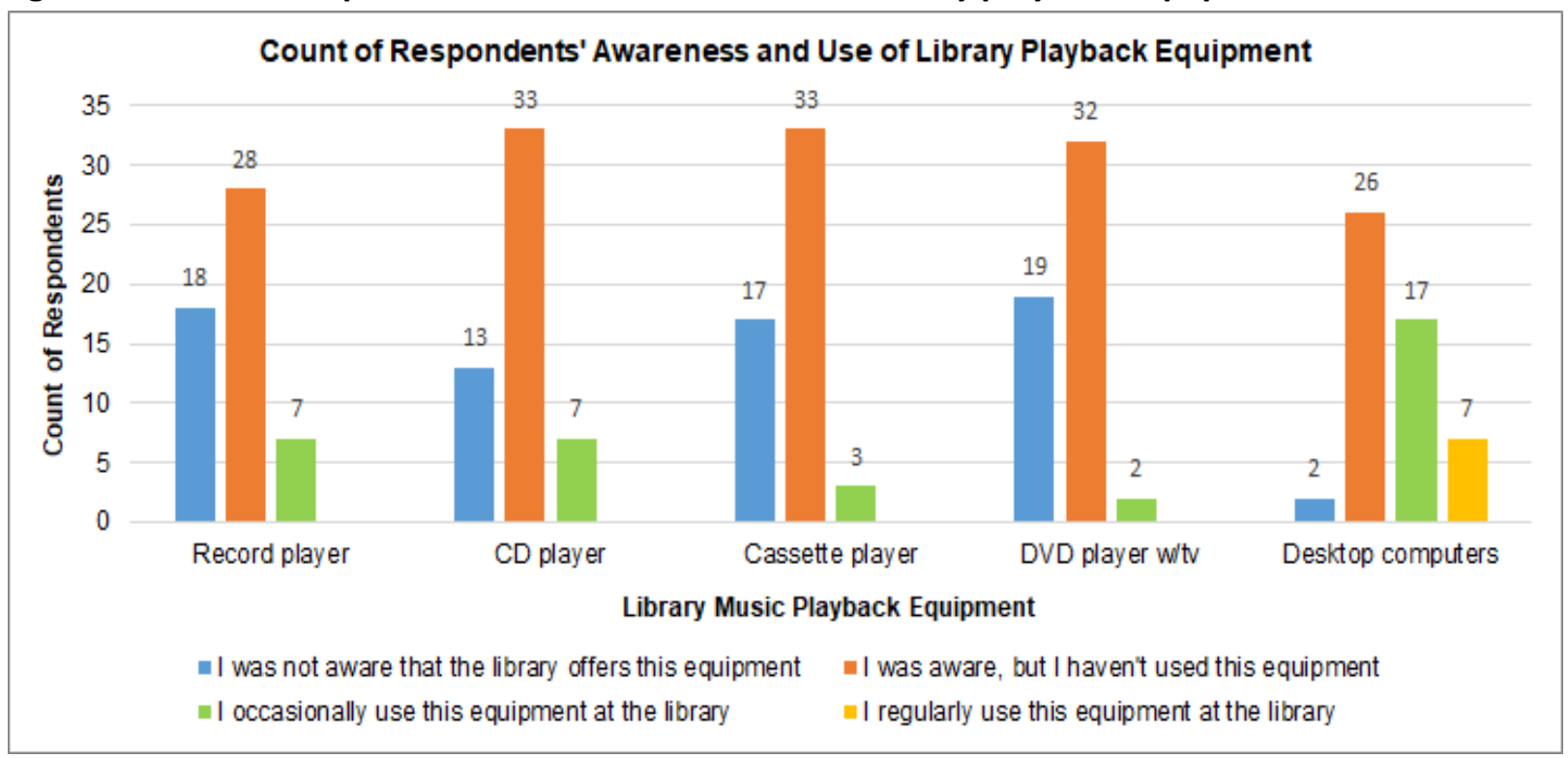


Q6) Why respondents do not use music formats and playback equipment available at UAL

When asked why they do not use the UAL music formats or playback equipment, some respondents indicated that they already owned the equipment or physical materials they needed. Several also referenced convenience associated with using their own equipment or materials and the inconvenience of borrowing physical materials from the library (i.e. none of the UAL's media are physically browsable; users must find items in the online catalogue and place holds on them). As well, the convenience of using online resources, whether the library's or publicly accessible platforms (i.e., YouTube, Spotify), eliminated much of the need to use legacy formats or playback machines.

Two respondents noted that although they did not need legacy format playback machines, they felt the library should still have them available for those who did. Four respondents also indicated that they did not know how to use legacy equipment, how to access physical library music formats, or reported fear or intimidation regarding the experience of going to the library or asking for help.

Section 3 - Library music resources: "What is important to you? What do you want?"

\section{Q7) Rated Importance of music formats, playback equipment and group listening spaces}

Figure 5 breaks down how respondents rated the importance of music formats from one (least important) to four (most important). While physical formats were rated as moderately important, respondents rated digital files and streaming audio as highly important.

Figure 5: Count of respondents' rated importance of library music formats

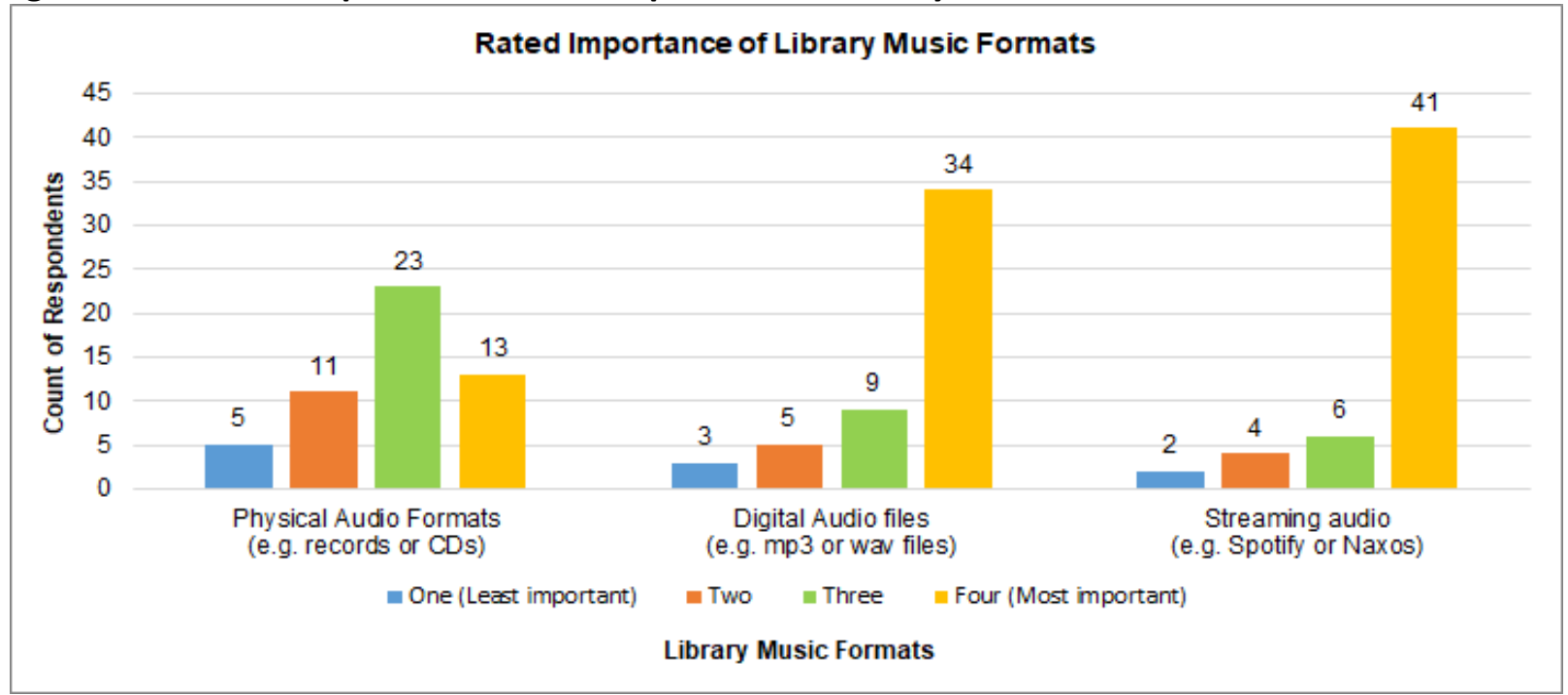


Feedback on the importance of circulating playback equipment and group listening space is represented in Figure 6. Circulating playback equipment was rated mostly as unimportant, though there was greater support for circulating mobile digital audio players. A group listening space was rated as more important than circulating playback equipment, but its rating was only moderately important overall.

Figure 6: Count of respondents' rated importance of circulating playback equipment and group listening spaces

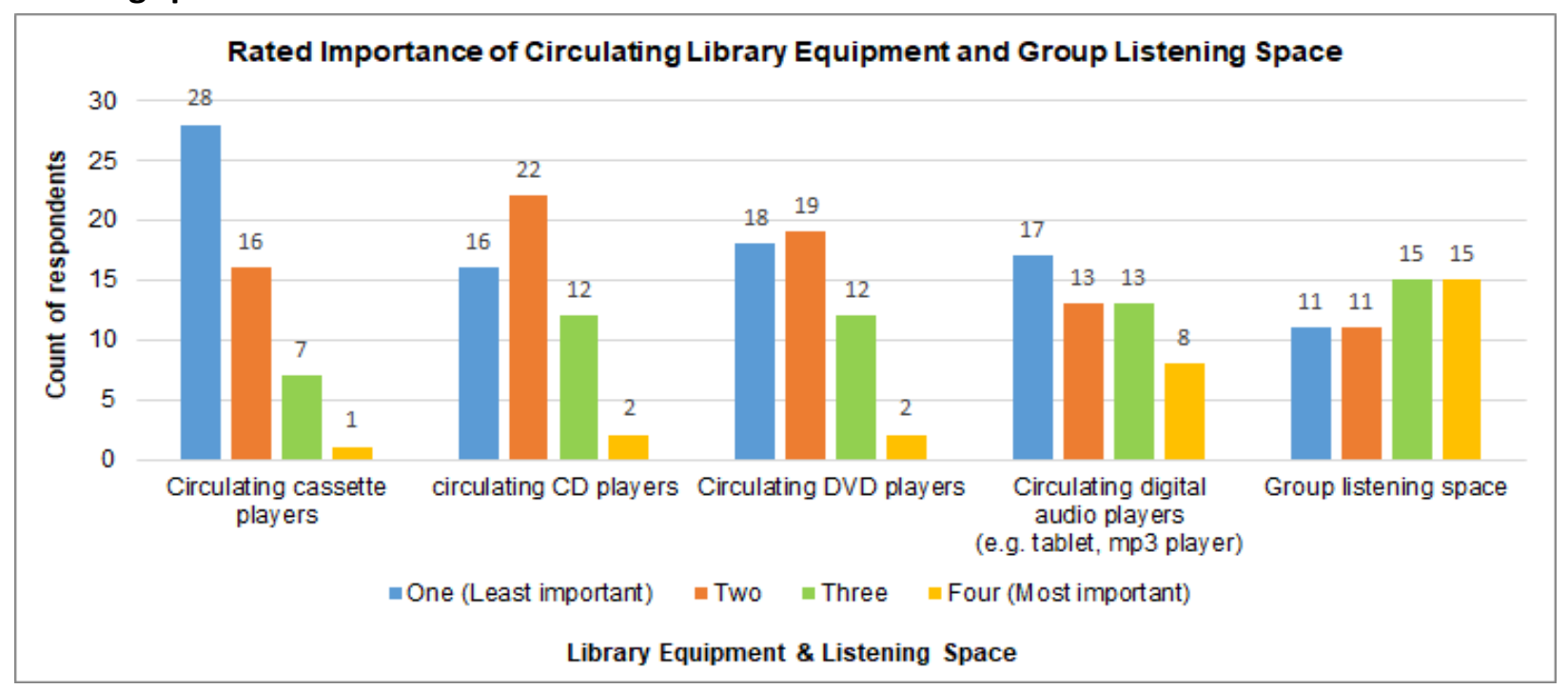

\section{Q8) Final feedback}

Only ten respondents provided additional feedback, and five of those responses included general statements of appreciation for the library and the services we provide. Specific suggestions for improvement, however, are paraphrased as follows:

- Don't eliminate physical media:

- DVDs are particularly important due to multichannel (surround) audio capabilities, and

- Physical media formats include valuable print information in the liner notes, as well as the album artwork.

- Provide various ways to listen to music, even if not everyone uses the older, more outdated equipment.

- Provide information about what kinds of listening tools are available and how to use them.

- Provide a truly high-quality listening space with surround sound, suitable for small groups as well as individual use.

- The library needs more resources for non-English music and world music cultures. 


\section{Discussion and Follow-up}

\section{Limitations}

The overall response rate for this survey, at $13.6 \%$, was fairly low and cannot be considered representative of the total MD population. The undergraduate students were particularly underrepresented in the survey results, and these findings may not be as reliable as the findings for the graduate student and staff/faculty populations. Additionally, not all respondents completed the entire survey.

Despite these limitations and the small data set, the results confirmed general impressions about how MD affiliates access music. They communicated a consistent message from each survey section: digital, broadcast and CD formats are regularly used and valued, as are library services that support access to these formats.

\section{Digital vs Physical Formats}

The survey results indicate that while most affiliates of the MD are aware of much that the UAL has to offer, the legacy formats are not used as highly as digital. This can likely be attributed to both the evolutionary trends of music technology as well as the general inconvenience of accessing physical formats and playback equipment at the UAL.

Table 3 shows that the total circulation of UAL physical music formats (LPs, cassettes, CDs and DVDs) is significantly lower compared to the combined total streaming events (for select streaming databases) for the calendar years 2017, 2018 and 2019. The vast majority of total circulations were $\mathrm{CDs}{ }^{7}$ which have shown a slight increase in use relative to overall circulation of physical music formats. This usage stands in contrast with overall decline in circulation and streaming activity as a whole.

It is important to note that streaming content usage (column 3) reflects individual tracks and will reasonably appear higher than physical format circulation numbers, as it is impossible to know how many tracks were used from the physical material formats. The trend of high CD circulations (compared to other physical media) and high streaming activity over the last three years also reflects the findings of survey question 2 which asked respondents to identify which music formats they most regularly use. As reported in Figure 1 (see page 4), streaming content and CDs were the second and third (respectively) most regularly used music formats after digital files (i.e. individual $\mathrm{mp3}$, mp4 files stored on individual devices) but which are not formats available through the UAL.

\footnotetext{
${ }^{7}$ Per circulation report run August 5, 2020.
} 
Table 3: Total circulation of physical music formats and Total CDs circulation, compared with streaming content usage for 2017, 2018, 2019

\begin{tabular}{|c|c|c|c|}
\hline Calendar year & $\begin{array}{l}\text { Total circulation of all physical } \\
\text { music formats (LPs, } \\
\text { cassettes, CDs and DVDs) }\end{array}$ & $\begin{array}{l}\text { Total CDs circulation } \\
\text { (\% of Total circulation) }\end{array}$ & $\begin{array}{l}\text { Combined Total Streaming } \\
\text { Events }^{8} \text { from } \text { Naxos }^{9} \text { and } \\
\text { Alexander Street }^{10}\end{array}$ \\
\hline 2017 & 1,930 & $1,685(87.3 \%)$ & 40,322 \\
\hline 2018 & 1,474 & $1,310(88.8 \%)$ & 34,293 \\
\hline 2019 & 964 & 869 (90.1\%) & 34,939 \\
\hline
\end{tabular}

\section{Physical Formats and Playback Equipment}

Despite the general trends reported by MD constituents for accessing music, information gathered from this survey still supports the ongoing acquisition of legacy formats (in particular CDs) and maintaining legacy playback equipment:

- CDs and other physical media are still circulating. Therefore, it stands to reason that we would provide a means of playing back this media to those who may not have this equipment at home.

- Many types of music outside the standard western canon (popular and classical) are not readily available through licensed streaming media. We will likely continue to acquire these in $C D$ format.

As indicated in the Survey Results section, respondents reported that they did not use music formats and playback equipment available at UAL due to their unfamiliarity with and fear of accessing resources offered by the library. This indicates that greater promotion of the library and its resources is needed for MD members to feel confident in using library resources.

In response to this and the comment "Provide information about what kinds of listening tools are available and how to use them," I revised the UAL Audio \& Video Resources Subject Guide ${ }^{11}$ so that students and staff/faculty have more information about the types of resources the UAL offers (both digital and physical) and how to access them. I have also incorporated promoting this guide in my library instruction sessions. The equipment available in Rutherford Library (i.e., my "home" library)

\footnotetext{
${ }^{8}$ Per usage reports run August 5, 2020 on selected streaming content providers: Naxos Music Library; Alexander Street Collections: Classical Music Library, Contemporary World Music, Dance in Video Vols 1-2, Jazz Music Library, American Music, Classical Music in Video, Classical Performance in Video, Opera in Video, Popular Music Library, Smithsonian Global Sound for Libraries

${ }^{9}$ Total number of tracks played.

${ }^{10}$ Number of successful full multimedia content unit requests.

${ }^{11}$ See UAL Guide here https://guides.library.ualberta.ca/audiovideo.
} 
is accompanied by a sheet with instructions for use, but I do not have any control over how Weir Library and Augustana Campus present their equipment for users.

\section{Response to Final Feedback and Future Plans}

The final feedback comments provided by respondents provided some clear directives for the UAL and demonstrated an interest in and concern for students' listening experiences. Regarding a group listening space, we were unable to "provide a truly high-quality listening space with surround sound", but we did install two large flat screen TV monitors equipped with Kramer ViaGo (a wireless presentation system) in two bookable study rooms. This permits small groups of up to 8-10 people to listen to digital music formats.

The comment "The library needs more resources for non-English music and world music cultures" highlighted two important issues regarding the music collection. Ongoing collection development must involve purposefully seeking out diverse voices for all music materials and formats. However, in addition to specific challenges associated with acquiring music resources that fall outside the Western canon, including distribution, licensing and format issues (the discussion of which fall outside the scope of this paper), the UAL faces further difficulties related to unprecedented fiscal cuts to higher education imposed by the Government of Alberta a few months after this survey was conducted. Despite these current challenges, discussions with the CSU are ongoing and I am hopeful we will be able to improve our collection in this regard.

This comment was also a welcome reminder that I needed to purposefully highlight the diverse voices in music resources that we already have. To that end, I have begun to modify the Music Subject Guide to feature music resources representing, for example, the music of non-western cultures and collections by composers who are Black, Indigenous, or Persons of Colour.

Initially, I had intended to conduct a follow up survey in April 2020, but the mandated closure of most in-person activity on our campus in mid-March due to the COVID-19 pandemic interrupted these plans and continues to affect library services. For example, the UAL originally stopped receiving new physical items into its collection, which will certainly impact our ability to expand non-western resources, at least in the short-term. And of course, the emphasis on digital access right now will likely affect the types of products that will be available ongoing. As well, with the projected long-term societal impacts of COVID-19, it's impossible to say if or when we might be able to offer access to community use equipment.

\section{Conclusion}

I created and designed this music listening survey in order to gain a better understanding of the music listening practices of MD affiliates at the University of Alberta and determine if the library was providing the resources and services needed to support these listening practices. The survey 
had some limitations, most notably that the responses were not fully representative of the MD population. However, it provided a clear picture of listening practices and provided valuable feedback for future collections acquisitions and library services at the University of Alberta Library.

\section{Appendix A: Music Listening Survey}

The purpose of this questionnaire is to help UAlberta Libraries understand your music listening practises and needs. We will use your responses to inform our collection development and improve services to meet your needs. Please note that completion of this survey is voluntary, and all information collected is anonymous.

\section{Section 1 of 3 - How do you listen to music?}

1. You are:

Undergrad Student

Graduate Student

Staff or Faculty

2. What are the formats you regularly use to listen to music? Select all that apply.

Vinyl records (any size)

Cassette tapes

CDs

O DVDs

Digital audio/video files (e.g. .mp3, .mp4, .wav)

Streaming audio/video (e.g. Spotify, Naxos)

Broadcast audio/video

Other:

Your answer

3. What equipment do you regularly use to listen to music? Select all that apply.

Record player

Cassette player

CD player 
DVD player

Digital player (e.g. tablet, smart phone, mp3 player)

Laptop or desktop computer

O Television/Radio

Other:

Your answer

\section{Section 2 of 3 - Library Music Resources}

4. Please tell us about your experiences with the following music formats offered by the library:

I was not aware that the

library offers this format
I was aware, but I haven't used this format
I occasionally use this

format from the library
I regularly use this format from the library

\begin{tabular}{|c|c|c|c|c|}
\hline Vinyl records & $\mathrm{O}$ & $\mathrm{O}$ & $\bigcirc$ & $\mathrm{O}$ \\
\hline CDs & $\bigcirc$ & $\mathrm{O}$ & $\bigcirc$ & 0 \\
\hline DVDs & $\bigcirc$ & $\bigcirc$ & $\mathrm{O}$ & S \\
\hline $\begin{array}{l}\text { Streaming } \\
\text { audio/video }\end{array}$ & $O$ & $\mathrm{O}$ & $O$ & \\
\hline
\end{tabular}

5. Please tell us about your experiences listening to music with the following "non-circulating" equipment offered by the library (i.e. equipment that must be used in the library):

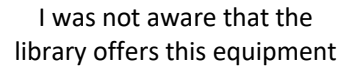
I was aware, but I haven't
used this equipment

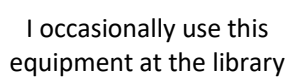

equipment at the library
I regularly use this equipment at the library
Record
player
O
$\bigcirc$
CD player
O
O
Cassette
player
O
DVD player
w/tv
O
Desktop
computers
$\bigcirc$

O

O

$O$

O

O

O

$O$ 
6. If you are aware of the library's formats and equipment, but do not regularly use them, please tell us what may have been preventing you from doing so.

\section{Your answer}

\section{Section 3 of 3 - Your feedback}

7. On a scale of $1-4$, with 1 the least important and 4 the most important, how important to you is each of the following?

\begin{tabular}{|c|c|c|c|c|}
\hline & 1 & 2 & 3 & 4 \\
\hline $\begin{array}{l}\text { Physical audio formats (e.g. } \\
\text { records or CDs) }\end{array}$ & $\mathrm{O}$ & $\bigcirc$ & $\mathrm{O}$ & $\mathrm{O}$ \\
\hline $\begin{array}{l}\text { Digital audio files (e.g. .mp3 or } \\
\text {.wav files) }\end{array}$ & $\mathrm{O}$ & $\bigcirc$ & $\mathrm{O}$ & $\mathrm{O}$ \\
\hline $\begin{array}{l}\text { Streaming audio (e.g. Spotify or } \\
\text { Naxos) }\end{array}$ & $\mathrm{O}$ & $\bigcirc$ & $\bigcirc$ & $\bigcirc$ \\
\hline Circulating cassette players & $\mathrm{O}$ & $\bigcirc$ & $\bigcirc$ & $\mathrm{O}$ \\
\hline Circulating CD players & $\mathrm{O}$ & $\bigcirc$ & O & $\mathrm{O}$ \\
\hline Circulating DVD players & $\mathrm{O}$ & $\mathrm{O}$ & $\bigcirc$ & $\mathrm{O}$ \\
\hline $\begin{array}{l}\text { Circulating digital audio players } \\
\text { (e.g. tablet, } \mathrm{mp} 3 \text { player) }\end{array}$ & $\mathrm{O}$ & $\mathrm{O}$ & $\mathrm{O}$ & \\
\hline Group listening space(s) & $\mathrm{O}$ & $\mathrm{O}$ & O & \\
\hline
\end{tabular}

8. Do you have any questions, or is there anything else you would like to tell us? Your answer 